\title{
Outcomes of ultrasound-guided percutaneous argon-helium cryoablation of hepatocellular carcinoma
}

\author{
Yongping Yang • Chunping Wang • Yinying Lu $\cdot$ Wenlin Bai $\cdot$ Linjing An • \\ Jianhui Qu $\cdot$ Xudong Gao $\cdot$ Yan Chen $\cdot$ Lin Zhou $\cdot$ Yu Wu Y Yongyi Feng • \\ Minna Zhang $\cdot$ Xiujuan Chang $\cdot$ Jiyun Lv
}

Published online: 21 December 2011

(C) The Author(s) 2011. This article is published with open access at Springerlink.com

\begin{abstract}
Purpose To evaluate the efficacy and safety of ultrasound (US)-guided percutaneous argon-helium cryoablation for hepatocellular carcinoma (HCC) and determine appropriate indications.

Methods We reviewed outcomes of $300 \mathrm{HCC}$ patients who underwent US-guided percutaneous cryoablation.

Results Overall, 223 tumors (mean diameter $7.2 \pm$ $2.8 \mathrm{~cm}$ ) in 165 patients were incompletely ablated, while 185 tumors (mean diameter $5.6 \pm 0.8 \mathrm{~cm}, P=0.0001$ vs. incomplete ablation) in 135 patients were completely ablated. Nineteen patients $(6.3 \%)$ developed serious complications while in hospital, including cryoshock syndrome in six patients, hepatic bleeding in five, stress-induced gastric bleeding in four, liver abscess in one and intestinal fistulas in one. Two patients died because of liver failure. The median follow-up was 36.7 months (range 6-63 months). The local tumor recurrence rate was $31 \%$, and was related to tumor size $(P=0.029)$ and tumor location $(P=0.037)$. The mean survival duration of patients with early, intermediate and advanced HCC (Barcelona Clinic Liver Cancer staging
\end{abstract}

C. Wang and Y. Yang contributed equally to this study.

Y. Yang $(\varangle) \cdot$ C. Wang · Y. Lu · W. Bai · L. An · J. Qu ·

X. Gao - Y. Chen · L. Zhou - Y. Wu · Y. Feng - M. Zhang ·

X. Chang . J. Lv $(\bowtie)$

Center of Therapeutic Research for Hepatocellular Carcinoma,

Beijing 302nd Hospital, Beijing 100039, China

e-mail: yongpingyang@hotmail.com

J. Lv

e-mail: 1vjy302@yahoo.cn system) was $45.7 \pm 3.8,28.4 \pm 1.2$ and $17.7 \pm 0.6$ months, respectively.

Conclusions US-guided percutaneous cryoablation is a relatively safe and effective therapy for selected HCC patients.

Keywords Cryoablation $\cdot$ Efficacy $\cdot$ Hepatocellular carcinoma $\cdot$ Safety $\cdot$ Treatment

\section{Introduction}

Hepatocellular carcinoma (HCC) is ranked as the third most common cause of cancer-related death worldwide [1] and as the second in China [2]. The resectability rate of HCC is limited to $20-30 \%$ of patients because of various factors such as multifocal disease and poor liver function reserve as a result of cirrhosis [3]. Liver transplantation is an alternative potentially curative treatment for early HCC, but is limited by the limited availability of donor organs. Hence, local ablative therapies, such as ethanol injection, radiofrequency ablation (RFA), laser photocoagulation, high-intensity focused ultrasound (US), microwave and cryoablation, have been proposed for the treatment of unresectable HCC.

Cryoablation is a technique based on in situ freezing and devitalization of tissues, which can be precisely controlled to produce a predictable zone of necrosis. Recently, Osada et al. [4] found that not only was the local tumor necrotic, but so was adjacent tumor tissue following cryoablation in HCC patients, which they regarded as ectopic tumor suppression. The technology has been used extensively in open surgical settings and, more recently, has been applied percutaneously to treat renal tumors [5]. However, other than in China, there are very few studies reporting the 
efficacy of ultrasound (US)-guided percutaneous cryoablation of HCC. Most of the bias against this percutaneous setting is based on a theoretical risk associated with an ablation modality that does not employ cauterization, such as heat-based ablation therapy, and because of the large probes, which can cause serious bleeding when removed [6]. However, experimental studies have found no significant difference in the rate of hemorrhage following ablation with a single RF probe versus a single cryoprobe [7]. Moreover, with the development of argon gas-based systems, the cryoprobes have decreased in size (now 15-17 gauge), meaning percutaneous cryoablation is now feasible. The percutaneous approach has two advantages over the laparotomic approach. First, the percutaneous approach is less invasive than laparotomic methods, an important consideration in terms of coagulation disorders that may lead to disseminated intravascular coagulation (DIC), liver failure and myoglobinuria, for example. Second, but most important, is that the ablation zone can be carefully monitored by US [4].

In this report, we document our experience of percutaneous cryoablation for $\mathrm{HCC}$ in a relatively large cohort of patients. The aim of this analysis was to demonstrate the safety and efficacy of percutaneous cryoablation, to assess the medium-term outcomes and evaluate the indications for this treatment modality.

\section{Materials and methods}

Patients

A total of 300 patients with $\mathrm{HCC}$ who were treated at the Center of Therapeutic Research for HCC, Beijing 302nd Hospital between April 2003 and June 2006 were included in this study. HCC was diagnosed based on imaging findings and $\alpha$-fetoprotein (AFP) levels, and was confirmed by needle core biopsy. The tumors were graded histologically based on Edmondson's grading system as follows: well differentiated, grade I or I-II; moderately differentiated, grade II or II-III; poorly differentiated, grade III or III-IV. Performance status (PS) was evaluated according to the Eastern Cooperative Oncology Group (ECOG) criteria. The Barcelona Clinic Liver Cancer (BCLC) classification [8] was used to identify tumor stage. The 300 patients had a total of 408 tumors of $1.9-15 \mathrm{~cm}$ in diameter (mean $6.4 \pm 3.3 \mathrm{~cm})$. The clinical characteristics of the patients are summarized in Table 1. All cryoablation treatments were approved by the Research Ethics Committee at Beijing 302nd Hospital, and written informed consent was obtained from all patients who met the inclusive criteria before collecting blood and tumor specimens, data collection and analysis.
Table 1 Patient characteristics

\begin{tabular}{ll}
\hline Clinical features & Values \\
\hline Gender, number (\%) & \\
Male & $256(85.3 \%)$ \\
Female & $44(14.7 \%)$ \\
Age (years), median (range) & $53(29-79)$ \\
Child-Pugh class, number (\%) & \\
A & $133(44.3 \%)$ \\
B & $167(55.7 \%)$ \\
Liver cirrhosis, number (\%) & \\
No & $68(22.7 \%)$ \\
Yes & $232(77.3 \%)$ \\
With portal invasion, number (\%) & $170(56.7 \%)$ \\
With inferior vena cava invasion, number $(\%)$ & $20(6.67 \%)$ \\
With HBV infection & $259(86.3 \%)$ \\
With HCV infection & $35(11.7 \%)$ \\
With HBV and HCV concurrent infection & $6(2 \%)$ \\
Tumor differentiation, number (\%) & \\
High & $56(18.7 \%)$ \\
Medium & $174(58.0 \%)$ \\
Low & $70(23.3 \%)$ \\
Tumor number & \\
1 & 208 \\
3 & 76 \\
BCLC stage, number (\%) & 16 \\
Intermediate & \\
Advanced & $34(11.3 \%)$ \\
\hline
\end{tabular}

$H B V$ hepatitis B virus, $H C V$ hepatitis $\mathrm{C}$ virus, $B C L C$ Barcelona Clinic Liver Cancer

\section{Inclusion and exclusion criteria}

Patients were included in this study if they met the following criteria: unresectable tumor because of the number and size of HCCs, tumor location and/or underlying illnesses that contraindicated surgery; were reluctant to undergo hepatic resection or liver transplantation; the HCC had recurred after resection; tumor metastasis with partial portal vein thrombosis (PVT) or vena cava invasion and the patient wished to destroy the tumor to reduce tumor load, even though they would not normally be considered for local ablative therapy; Child-Pugh class A or B liver function, with a serum bilirubin level $<51.3 \mu \mathrm{mol} / \mathrm{l}$; and ECOG PS $\leq 2$. Patients were excluded for the following reasons: BCLC stage D (ECOG $\mathrm{PS}>2$, Child-Pugh $\mathrm{C}$ ); obvious cirrhotic ascites; had tumor thrombosis at the main branch of the portal vein, and the size of the thrombosis 
exceeded $50 \%$ of the diameter of the portal vein; extrahepatic metastases in the lung, bone or brain; or use of other ablative therapies or TACE before cryoablation.

\section{Treatment selection}

Patients with a tumor $<5 \mathrm{~cm}$ in diameter were intended to receive curative therapy with single or repeated cryoablation. Patients with incomplete ablation or tumors $\geq 5 \mathrm{~cm}$ underwent transarterial chemoembolization (TACE) after cryoablation. For patients with partial PVT or vena cava invasion, based on the patient's wishes, liver function and financial status, cryoablation and TACE were performed in combination with systemic therapy with the multikinase inhibitor sorafenib. For TACE, super-selective catheterization was performed directly to the artery supplying the tumor, wherever possible, to avoid hepatic failure and severe adverse events.

\section{Preoperative preparation}

The coagulation profile, including prothrombin time, bleeding time, coagulation time and plasma biochemical assay, was determined for each patient before the procedures. Oral anticoagulants were stopped for 3 days before the operation. Patients who coughed frequently were given an antitussive. The blood pressure of hypertensive patients was controlled to approximately $140 / 90 \mathrm{mmHg}$, and the blood glucose levels of diabetic patients were lowered to $<10 \mathrm{mmol} / \mathrm{l}$. All patients were injected with $10 \mathrm{mg}$ morphine and $10 \mathrm{mg}$ of an antipsychotic before entering the operating room. An intravenous infusion route was also established immediately before the procedure.

\section{Argon-helium cryoablation procedure}

Argon-helium cryoablation was performed as previously described [9]. Briefly, an argon-helium gas-based CRYOcare system (EndoCare, Irvine, CA, USA) and cryoprobes were used to freeze the tumor with a dual freeze-thaw cycle under ultrasound guidance. After sonographically determining the most favorable percutaneous approach, we inserted the cryoprobes into the tumor under US guidance and advanced the tip to reach the distal margin of the targeted lesion. The number of probes used depended on the location and size of the lesions to be ablated. The dual freeze-thaw cycle was comprised of a 20-min freeze, followed by a 10-min thaw and a 15-min freeze. The dimensions of the frozen tissue were monitored by US. The cryoprobe temperatures were reduced with $1 \mathrm{~min}$ to $-135 \pm 2{ }^{\circ} \mathrm{C}$. Upon removal of the probes, all tracts were packed with Surgicel (Johnson \& Johnson, Inc., Arlington, TX, USA) to control bleeding. We aimed at curative ablation of all tumors by leaving a 5-mm margin beyond the edge of the tumor, wherever feasible, in a single cryoablation. The procedure was performed under conscious sedation. The cardiogram, ventilation and oxygen saturation levels were monitored throughout. Warming mats were used to keep the patients warm during the cryoablation.

\section{Postoperative management}

After treatment, all patients were observed in an intensive care unit overnight to monitor for life-threatening delayed hemorrhage and other complications. Patients received analgesic drugs for pain control, as well as antiemetic therapy and sedatives as necessary. Intravenous fluid replacement was also conducted. Mannitol was used to induce osmotic diuresis, and aminocaproic acid was infused (5-g loading dose followed by $1 \mathrm{~g} / \mathrm{h}$ for $6 \mathrm{~h}$ ) to reduce coagulopathy. We also infused $5 \%$ sodium bicarbonate $(40-60 \mathrm{ml})$ intravenously to maintain urine at $\mathrm{pH}$ $>6$ and urine output at $>100 \mathrm{ml} / \mathrm{h}$. In addition, hemostatic, antibiotic (third-generation cephalosporin or third-generation fluoroquinolone antibacterial agents) and nutrition support (blood plasma $200 \mathrm{ml} /$ day or human albumin $10 \mathrm{~g} /$ day) therapies were provided for 5 days after cryoablation.

\section{Follow-up}

Helical contrast computed tomography (CT) or magnetic resonance imaging (MRI) scans were done 1 week after cryoablation to determine the extent of ablation. Patients with incomplete ablation underwent a second or third session of cryoablation to completely ablate the tumor or TACE using lipiodol plus $10-20 \mathrm{mg}$ epirubicin hydrochloride. All patients were followed and underwent serial monitoring of serum AFP, chest radiographs, and CT or MRI scans every 2 months to detect tumor recurrence. Local recurrence was defined as tumor recurrence within or at the periphery of the ablated lesion. Distant intrahepatic recurrence was defined as a new tumor that appeared in a region of the liver separate from the ablated area. Extrahepatic recurrence refers to any tumor recurrence outside the liver.

\section{Statistical analysis}

Comparisons between groups were done using $\chi^{2}$ tests. Local tumor recurrence was analyzed by logistic regression. Survival rates were estimated by the Kaplan-Meier method and compared using the log rank test. All analyses were done using SPSS software version 13 (SPSS Inc., Chicago, IL, USA). $P$ values $<0.05$ were considered statistically significant. 


\section{Results}

Patients and procedures

A total of 559 cryoablations were performed in 408 tumors in 300 patients with HCC, with 1, 2, 3, 4 and 8 procedures in 112, 129, 51, 7 and 1 patient, respectively. CT scanning showed that that 223 tumor lesions with a diameter of $5.0-15 \mathrm{~cm}$ (mean $7.2 \pm 2.8 \mathrm{~cm}$ ) in 165 patients were not completely ablated, while 185 tumor lesions with a diameter of $1.9-7.0 \mathrm{~cm}(5.6 \pm 0.8 \mathrm{~cm})$ in 135 patients were completely ablated. Liver function and complications were evaluated in all of the patients. This included 35 patients who were discharged from the hospital 2-3 weeks after incomplete ablation, but who were lost to follow-up because of incorrect contact details. These 35 patients developed no severe complications in the hospital. The remaining 265 patients were followed up for a median of 36.7 months (range 6-63 months).

Mortality and adverse events

The cryoablation-related adverse events were observed during and after treatment. Percutaneous cryoablation caused transient elevations in alanine aminotransferase and bilirubin levels, and mildly decreased platelet (PLT) levels, which returned to baseline levels about 2 weeks after treatment, at which time they were not significantly different from the levels before cryoablation $(P>0.05$, Table 2). However, severe liver dysfunction occurred in seven patients, two of whom had ascites. After treatment for 30-45 days, five patients recovered and two died of liver failure. The seven patients belonging to Child-Pugh class B (score $>8$ ) and together with PVT were considered as having advanced HCC. In these seven patients, the mean total estimated area (TEA) of the tumor to be cryoablated was $70 \%$ (Table 3).

Severe complications occurred in 19 patients $(6.3 \%)$. Five of these $(1.7 \%)$ developed liver hemorrhage, which was usually diagnosed within 3-6 h after the cryoablation. In one of these patients, there was a lesion in the caudate lobe of the liver, while the tumors in the other four patients were near the liver capsule but were not surrounded by liver parenchyma (Fig. 1). In one patient, using CT, we detected a subcapsular hemorrhage that extended from the ablation zone. Fortunately, the liver capsule was intact, and the patient recovered following transcatheter arterial embolization (TAE) and supportive measures. The other

Table 2 Effects of percutaneous argon-helium cryoablation for HCC on liver function parameters

\begin{tabular}{lcccc}
\hline Liver function & Preoperation & Postoperation 1 day & Postoperation 7 days & Postoperation 14 days \\
\hline ALT $(\mathrm{U} / \mathrm{l})$ & $57.8 \pm 21.7$ & $193.1 \pm 122.7^{*}$ & $72.7 \pm 65.3^{*}$ & $46.9 \pm 21.13$ \\
TBIL $(\mu \mathrm{mol} / \mathrm{l})$ & $23.7 \pm 18.9$ & $45.5 \pm 39.8^{*}$ & $28.23 \pm 19.7^{*}$ & $29.4 \pm 25.6$ \\
TP $(\mathrm{g} / \mathrm{l})$ & $66.8 \pm 6.3$ & $65.7 \pm 5.1$ & $63.2 \pm 8.7^{*}$ & $65.4 \pm 7.2^{*}$ \\
ALB $(\mathrm{g} / \mathrm{l})$ & $37.6 \pm 3.9$ & $36.5 \pm 4.6$ & $35.4 \pm 6.3^{*}$ & $32.0 \pm 5.9 *$ \\
PA $(\%)$ & $81.1 \pm 12.0$ & $78.5 \pm 13.6$ & $78.4 \pm 21.5$ & $82.8 \pm 19.1$ \\
PLT $\left(\times 10^{9} / 1\right)$ & $126.2 \pm 83.2$ & $113.5 \pm 81.4 *$ & $127.3 \pm 83.7$ & $127.8 \pm 84.7$ \\
\hline
\end{tabular}

$A L T$ alanine aminotransferase, $T B I L$ total bilirubin, $T P$ serum total protein, $A L B$ serum albumin, $P A$ prothrombin activity, $P L T$ platelet

$* P<0.01$ versus before operation

Table 3 Liver function and total estimated area (TEA) of cryoablation in seven HCC patients who developed severe liver damage following cryoablation

\begin{tabular}{|c|c|c|c|c|c|c|c|c|c|c|c|c|}
\hline \multirow[t]{2}{*}{ Patient } & \multicolumn{2}{|c|}{ Child-Pugh class } & \multicolumn{2}{|c|}{ CHE (g/l) } & \multicolumn{2}{|c|}{ ALB (g/l) } & \multicolumn{2}{|c|}{ TBil $(\mu \mathrm{mol} / \mathrm{l})$} & \multirow{2}{*}{$\begin{array}{l}\text { Tumor } \\
\text { diameter }(\mathrm{cm})\end{array}$} & \multirow{2}{*}{$\begin{array}{l}\text { Portal vein } \\
\text { invasion }\end{array}$} & \multirow[t]{2}{*}{ TEA (\%) } & \multirow[t]{2}{*}{ Prognosis } \\
\hline & Pre & Post & Pre & Post & Pre & Post & Pre & Post & & & & \\
\hline 1 & $\mathrm{~B}(8)$ & $\mathrm{C}(11)$ & 4948 & 3067 & 36 & 31 & 61.7 & 143.9 & 10 & Yes & 70 & Improved \\
\hline 2 & $\mathrm{~B}(9)$ & $\mathrm{C}(10)$ & 1620 & 1683 & 30 & 30 & 44.1 & 138.5 & 9 & Yes & 60 & Improved \\
\hline 3 & $\mathrm{~B}(9)$ & $\mathrm{B}(7)$ & 3552 & 3601 & 27 & 42 & 83.7 & 214.4 & 8 & Yes & 80 & Improved \\
\hline 4 & $\mathrm{~B}(8)$ & $\mathrm{C}(10)$ & 4415 & 3601 & 41 & 38 & 35.7 & 218.5 & 12 & Yes & 70 & Died \\
\hline 5 & $\mathrm{~B}(9)$ & $\mathrm{C}(15)$ & 4107 & 2981 & 33 & 28 & 38.5 & 79.6 & 12 & Yes & 70 & Improved \\
\hline 6 & $\mathrm{~B}(9)$ & $\mathrm{C}(15)$ & 4107 & 2981 & 33 & 28 & 38.5 & 339.3 & 11 & Yes & 70 & Died \\
\hline 7 & $\mathrm{~B}(8)$ & $\mathrm{B}(9)$ & 4500 & 3600 & 33 & 32 & 35.4 & 94.3 & 10 & Yes & 70 & Improved \\
\hline
\end{tabular}

Pre pretreatment, Post posttreatment, $C H E$ cholinesterase, ALB serum albumin, TBIL serum total bilirubin 
four patients showed hepatorrhexis bleed into the peritoneal cavity. The five patients with liver bleeding were cured by immediate TAE. Two patients $(0.7 \%)$ developed liver failure (Table 3) and died at 35 and 45 days, respectively.

Six patients who underwent cryoablation and had TAE of $50-60 \mathrm{~cm}^{2}$ developed cryoshock syndrome with chills, fever, tachycardia, tachypnea and temporary renal damage. All six patients recovered following intravenous atropine and 5\% sodium bicarbonate, being covering with an electric blanket and oxygen inhalation. Four patients (1.3\%) developed stress-related gastric mucosal lesion-induced hemorrhage, and they were treated with acid-inhibiting and gastric mucosa-protecting agents. One patient developed a liver abscess at the ablation zone that was not absorbed, and the abscess content exuded from a scar of the probe tract 1 month after cryoablation and required drainage

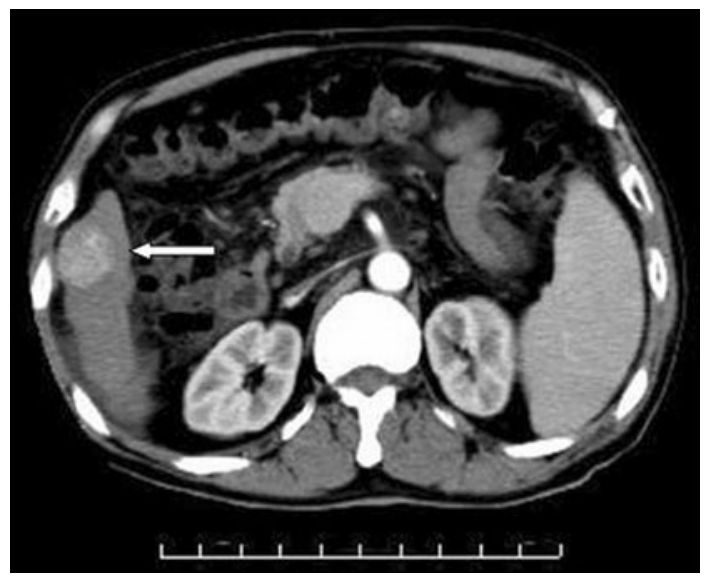

Fig. 1 The location of the tumor in a patient with liver hemorrhage after cryoablation, which was close to the liver capsule without being surrounded by liver parenchyma (solid arrows)

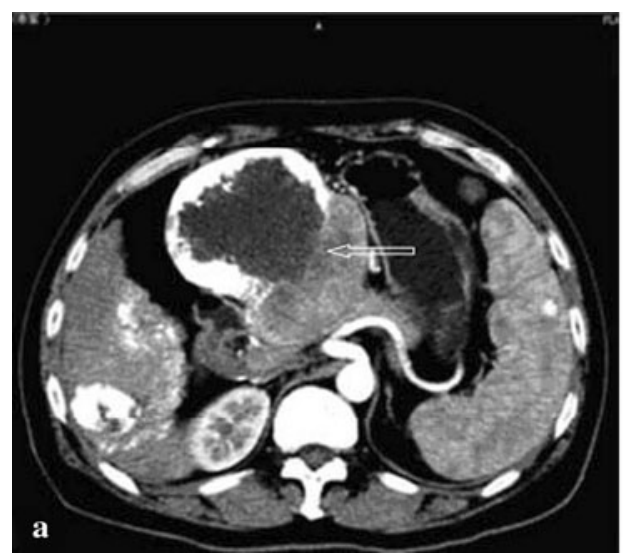

Fig. 2 Liver abscess following cryoablation. The liver abscess occurred in the ablation zone and was not absorbed. The abscess content exuded from a scar of a probe tract 1 month after cryoablation
(Fig. 2). In one patient, the loop of the bowel was injured and intestinal fistulas formed, which required surgical repair (Fig. 3). Minor complications were also noted, including pneumothorax, hemothorax, pleural effusion and postablation syndrome (e.g., fever, pain, skin frostbite).

Follow-up and survival

Of the 300 patients, 35 were discharged from the hospital without experiencing severe complications, but we could not follow-up these patients because of incorrect contact details. Therefore, 265 patients were included in our follow-up, including 135 with complete ablation and 130 patients with incomplete ablation. Among those with incomplete ablation, 87 underwent postoperative TACE (median 2.0 times, range 1-4 times) and 43 received a multikinase inhibitor (sorafenib). The median duration of follow-up was 36.5 months (range 6-63 months). Overall, 168 of 265 patients died for the following reasons (Table 4): esophagogastric variceal bleeding in 61 (36.3\%), liver failure in $44(26.2 \%)$, tumor recurrence/metastasis in $39(23.2 \%)$, tumor rupture and hemorrhage in 15 (8.9\%), and refractory ascites-induced renal failure in nine $(5.4 \%)$.

The overall 1-, 2- and 3-year survival rates were 80, 45 and $32 \%$, respectively, with a mean survival of 28 months and median survival of 22 months. The mean survival of patients with early HCC was $45.7 \pm 3.8$ months, and their 1-, 2- and 3-year survival rates were 91,85 and $65 \%$, respectively. The respective values in intermediate HCC were $28.4 \pm 1.2$ months, 87,62 and $45 \%$, respectively, and those in advanced HCC were $17.7 \pm 0.6$ months, 73, 25 and $12 \%$, respectively (Fig. 4). The mean recurrence-free survival of patients with early HCC was $39.7 \pm 3.8$ months, and their 1-, 2- and 3-year recurrence-free survival rates were 91,79 and $53 \%$, respectively. The respective values in

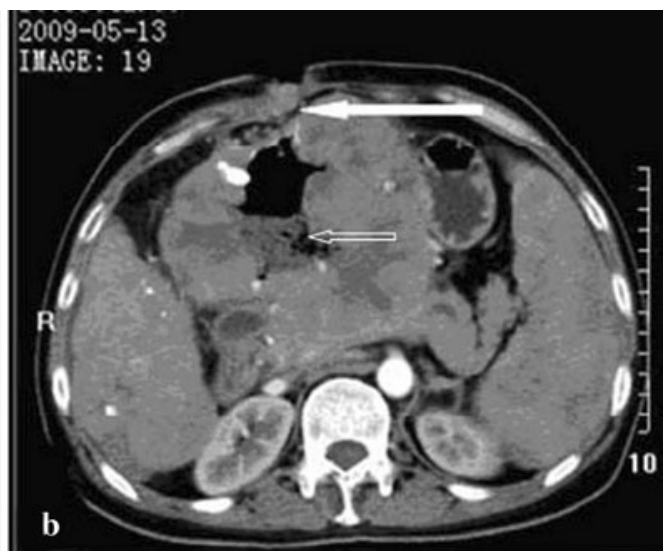

and required drainage. a The tumor before cryoablation (open arrow). b A sinus (solid arrow) formed between the skin and the liver abscess 

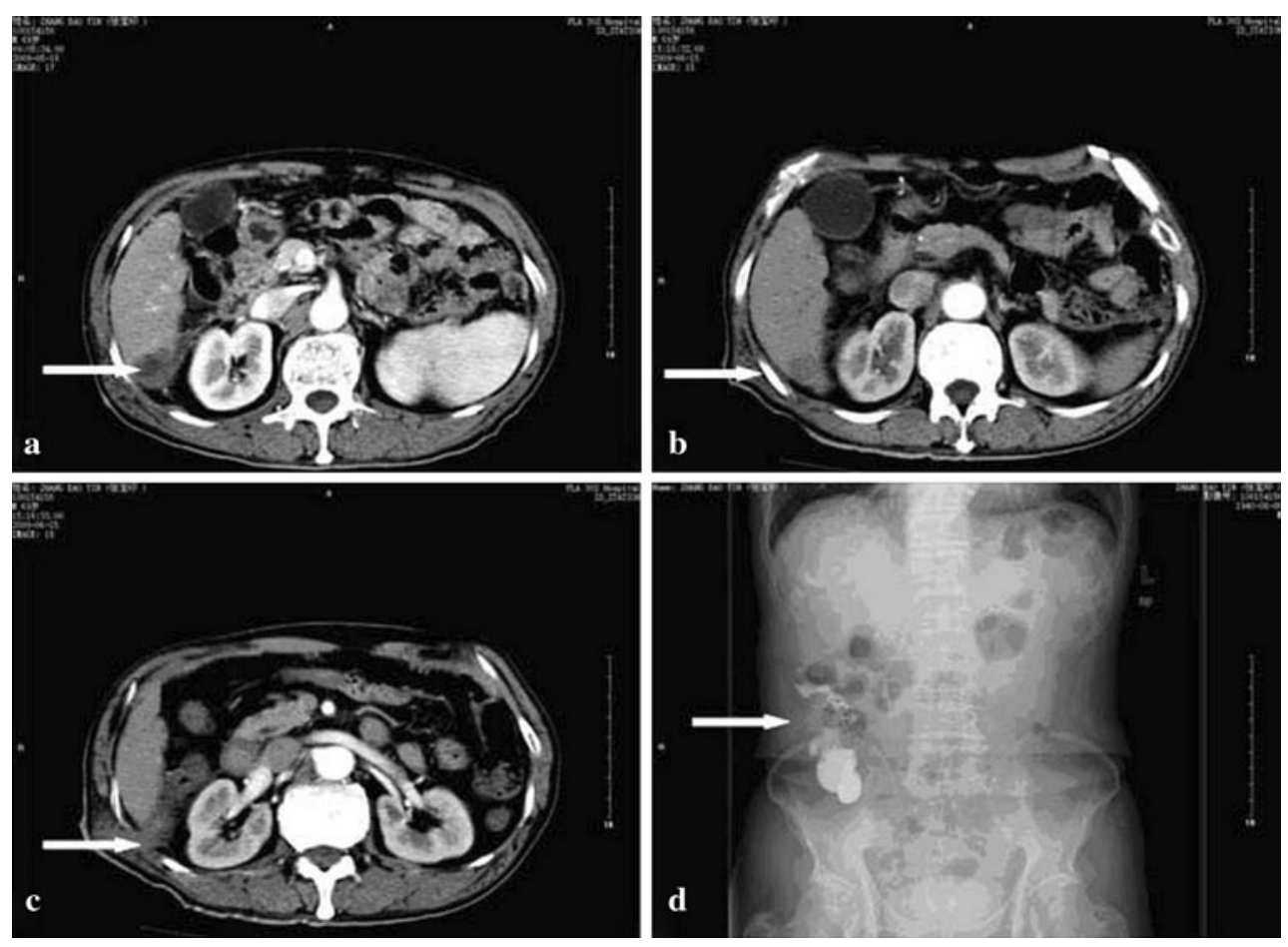

Fig. 3 Bowel loop injury and formation of intestinal fistulas following cryoablation. a The tumor before cryoablation. b The

tumor was completely ablated and decreased in size. c The intestinal fistulas formed 1 month after cryoablation. d The intestinal fistulas were confirmed on X-ray images following iohexol administration

Table 4 Causes of death in 168 patients after cryoablation according to tumor stage

\begin{tabular}{llll}
\hline Causes & $\begin{array}{l}\text { Early } \\
(n)\end{array}$ & $\begin{array}{l}\text { Intermediate } \\
(n)\end{array}$ & $\begin{array}{l}\text { Advanced } \\
(n)\end{array}$ \\
\hline Esophagogastric variceal bleeding & 4 & 17 & 40 \\
Liver failure & 2 & 12 & 30 \\
Tumor recurrence and metastasis & 0 & 13 & 26 \\
Tumor rupture and hemorrhage & 0 & 4 & 11 \\
$\begin{array}{l}\text { Refractory ascites-induced renal } \\
\quad \text { failure }\end{array}$ & 2 & 1 & 6 \\
Total & 8 & 47 & 113 \\
\hline
\end{tabular}

intermediate HCC were $21.4 \pm 1.5$ months, 68, 41 and $24 \%$, respectively. For advanced HCC, the median progressfree survival was 7.1 months (Fig. 5).

\section{Tumor recurrence}

Tumors recurred in 190 of 265 patients, with intrahepatic recurrence in 156 patients, intrahepatic plus extrahepatic recurrence in 26 patients and extrahepatic recurrence alone in 8 patients. Among 182 patients with intrahepatic recurrence, 106 developed local recurrence, whereas the other 76 patients developed distant intrahepatic recurrence. For the 34 patients with extrahepatic recurrence, 16 had pulmonary metastasis, 10 had retroperitoneal or celiac

lymph node metastasis, 3 had bone metastasis and 5 had adrenal metastasis. There was no evidence of needle-track metastasis.

\section{Local recurrence}

Local recurrence after cryoablation remains one of the main limitations of this therapy. Overall, 185 lesions in 135 patients with complete cryoablation and 102 lesions in 87 patients with incomplete cryoablation followed by TACE were considered effectively destroyed. Of these, 89 lesions (of 287, 31\%) recurred at the site of cryoablation. Several tumor factors possibly influence the local recurrence, including tumor size and location, tumor stage, tumor differentiation and mode of therapy. The rate of local recurrence increased with increasing tumor size, with rates of $7.1 \%$ (4/56), $25.5 \%$ (26/102) and $45.7 \%$ (59/129) for tumors with a diameter of $<3,3-5$ and $>5 \mathrm{~cm}$, respectively. The rates of local recurrence was significantly lower in tumors with complete ablation versus those with incomplete ablation followed by TACE [15.1\% (28/185) vs. $59.8 \%$ (61/102), respectively; $P=0.0001]$.

Sixty-seven of the 287 tumors were located in segments 4,5 and 8 , and were thus close to the inferior vena cava or portal vein with large venous affluxes. Tumor recurred in $41(61.2 \%)$ of these tumors (Table 5). Tumor differentiation and tumor stage were not associated with local 
Fig. 4 Overall survival (a) and prognostic influence of BCLC stage on overall survival rate after cryoablation in the 265 patients (b)
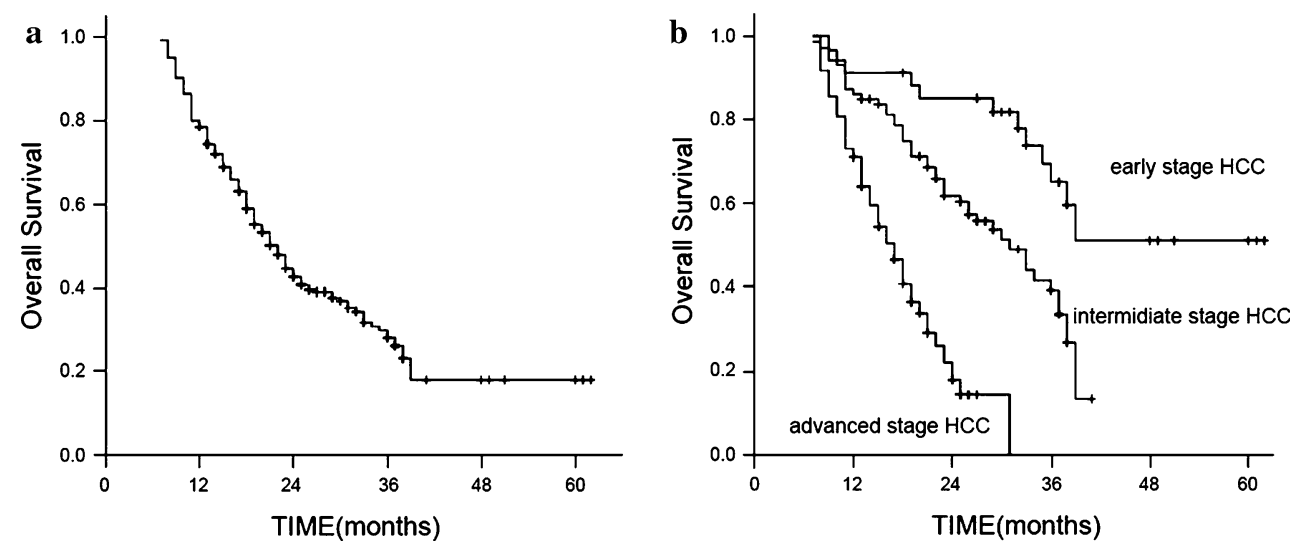

Fig. 5 Prognostic influence of BCLC (early stage HCC and intermediate stage HCC) a on recurrence-free survival and advanced $\mathrm{HCC} \mathbf{b}$ on progressfree survival
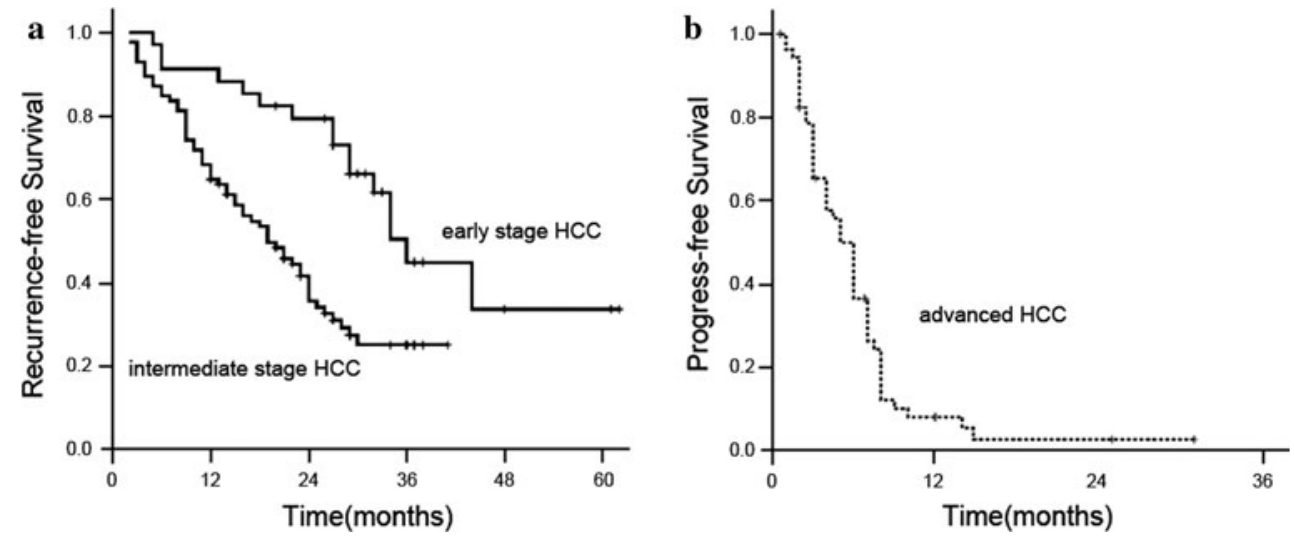

Table 5 Univariate analysis of factors associated with local recurrence

BCLC Barcelona Clinic Liver Cancer, TACE transarterial chemoembolization, $V C$ vena cava

\begin{tabular}{lll}
\hline Factors & $\begin{array}{l}\text { No. of local } \\
\text { recurrence }(\%)\end{array}$ & $P$ value \\
\hline BCLC stage (tumors/no. of patients) & $13(23.2)$ & \\
Early (56/34) & $46(36.5)$ & \\
Intermediate (126/86) & $33(28.6)$ & 0.0001 \\
Advanced (105/102) & & \\
Tumor size (no. of tumors) & $4(7.1)$ & 0.3928 \\
$<3$ cm (56) & $26(25.5)$ & \\
$3-5$ cm (102) & $59(45.7)$ & \\
$>5$ cm (129) & $12(23.1)$ & \\
Tumor differentiation (no. of tumors) & $44(32.8)$ & \\
High (52) & $33(32.7)$ & \\
Medium (134) & & 0.0001 \\
Low (101) & $28(15.1)$ & \\
Therapy method (no. of tumors) & $61(59.8)$ & \\
Complete cryoablation (185) & & \\
Incomplete cryoablation plus TACE (102) & $41(61.2)$ & \\
Tumor location (no. of the tumors) & $48(21.8)$ & \\
Close to VC or portal vein (67) &
\end{tabular}


Table 6 Multivariate analysis of factors associated with local recurrence

\begin{tabular}{lll}
\hline Variable & Risk ratio (95\% confidence interval) & $P$ \\
\hline BCLC stage & $0.782(0.142-1.568)$ & 0.220 \\
Tumor differentiation & $1.059(0.707-1.649)$ & 0.724 \\
Methods of therapy & $0.523(0.182-1.506)$ & 0.230 \\
Tumor size & $2.120(1.082-4.513)$ & 0.029 \\
Tumor location & $1.956(1.042-3.671)$ & 0.037 \\
\hline
\end{tabular}

BCLC Barcelona Clinic Liver Cancer

Table 7 Comparisons of clinical characteristics between patients with complete and incomplete ablation

\begin{tabular}{|c|c|c|c|}
\hline Factors & $\begin{array}{l}\text { Complete } \\
\text { ablation }(n)\end{array}$ & $\begin{array}{l}\text { Incomplete } \\
\text { ablation }(n)\end{array}$ & $P$ \\
\hline Gender & & & 0.9477 \\
\hline Male & 115 & 141 & \\
\hline Female & 20 & 24 & \\
\hline Age (years) & $51 \pm 14$ & $55 \pm 10$ & 0.079 \\
\hline Cirrhosis & 104 & 128 & 0.9117 \\
\hline Tumor differentiation & & & 0.8329 \\
\hline High & 27 & 29 & \\
\hline Medium & 78 & 96 & \\
\hline Low & 30 & 40 & \\
\hline Cryoablation (number) & & & 0.123 \\
\hline 1 & 43 & 69 & \\
\hline 2 & 67 & 62 & \\
\hline 3 & 23 & 28 & \\
\hline More & 2 & 6 & \\
\hline Tumor diameter & $5.6 \pm 0.8$ & $7.2 \pm 2.8$ & 0.0001 \\
\hline Child-Pugh classification & & & 0.972 \\
\hline A & 60 & 73 & \\
\hline B & 75 & 92 & \\
\hline Tumor no. & & & 0.8904 \\
\hline 1 & 92 & 116 & \\
\hline 2 & 36 & 40 & \\
\hline 3 & 7 & 9 & \\
\hline Portal or vena cana invasion & 70 & 100 & 0.1279 \\
\hline
\end{tabular}

TACE transarterial chemoembolization

recurrence. On the other hand, multivariate analysis (Table 6) showed that tumor size and tumor location were independent factors associated with local recurrence $(P=0.029$ and 0.037 , respectively).

Association between tumor diameter and complete ablation

CT scans showed that 223 tumors (mean diameter $7.2 \pm 2.8 \mathrm{~cm}$, range $5.0-15 \mathrm{~cm}$ ) in 165 patients were incompletely ablated, while 185 tumors (mean diameter $5.6 \pm 0.8 \mathrm{~cm}$, range $1.9-7.0 \mathrm{~cm}$ ) in 135 patients were completely ablated. There were no significant differences in terms of sex, age, presence of liver cirrhosis, tumor differentiation, number of cryoablation sessions, ChildPugh class, portal or vena cava invasion, or number of tumors between these two groups of patients $(P>0.05)$. However, the tumor diameter was significantly different between the two groups of patients $(t=5.65, P=0.0001$; Table 7).

\section{Discussion}

Argon-helium cryoablation is a new local ablative modality for the treatment of tumors. Cryotherapy is believed to kill cells by several mechanisms, including intracellular ice formation, solute-solvent shifts that cause cell dehydration and rupture, small vessel obliteration causing hypoxia, and specific antitumor immunoreactions that limit tumor growth [4, 10-12]. Currently, however, few studies outside of China have evaluated US-guided percutaneous cryoablation. In comparison, several research groups in China have tested percutaneous cryoablation. Indeed, two recent studies have described the outcomes of percutaneous cryoablation for HCC under CT or MRI guidance, and showed that it was safe and effective $[4,13]$. In one of these studies, the complete ablation rate for HCC of $<5 \mathrm{~cm}$ in diameter was $80.8 \%$ at 3 years [13].

In this study, our aim was to evaluate the efficacy and complications associated with this modality, and evaluate the indications for US-guided percutaneous cryoablation of HCC. Therefore, we included many patients in this study, even those who would not normally be candidates for local ablative therapy. In recent years, numerous studies of local ablation in combination with systemic therapy for advanced HCC have been performed. Therefore, we performed local tumor cryoablation for the majority of patients with vascular invasion under the condition that they wanted this treatment, and received it in combination with TACE or systemic therapy. Moreover, there was a large number of patients with early disease that would normally be considered for radical therapy, such as resection and transplantation, because these patients had a history of mild to severe chronic liver dysfunction because of hepatitis $\mathrm{B}$ or $\mathrm{C}$ infection, and are at high risk of secondary growths of new HCC $[14,15]$, and did not want to undergo hepatic resection and transplantation.

Based on the results of this study, we suggest that patients with a tumor of $<5 \mathrm{~cm}$ in diameter are suitable for percutaneous cryoablation. Tumor diameter is related to adequate ablation and local tumor recurrence. The mean diameter of the tumors with complete ablation was $5.6 \mathrm{~cm}$, 
and the rate of local recurrence was higher in tumors with a diameter $>5 \mathrm{~cm}$. Several reasons for this may exist, including the difficulty in ablating larger tumors because of inadequate freezing margins and the proximity of larger tumors to vascular structures resulting in incomplete cryoablation. In vivo experiments showed that the 3-mm probe could produce an iceball of $3 \mathrm{~cm}$ in diameter. Therefore, while tumors $<3 \mathrm{~cm}$ in diameter can be treated with a single 3 -mm cryoprobe, tumors with a diameter of $3-5 \mathrm{~cm}$ or $>5 \mathrm{~cm}$ may require $2-3$ and 3-4 cryoprobes, respectively. Theoretically, if multiple probes can be placed simultaneously, very large ablation zones can be achieved. However, in actual clinical practice, using more than three $3-\mathrm{mm}$ probes simultaneously in a percutaneous setting under US guidance is technically challenging. First, the 3-mm cryoprobe is relatively large and, in most cases, percutaneous cryoablations were performed via the intercostal approach, which limits the number of probes that can be used simultaneously. Second, although there are many advantages of US to monitor the extent of freezing, including its excellent soft tissue resolution, as well as providing real-time feedback regarding tumor location and freezing, incomplete imaging of the entire frozen tissue, low spatial resolution in deeper structures and operator dependency are disadvantages of the technique. The frozen tissue is identified as a hyperechoic boundary with dense posterior shadowing, which allows excellent visualization of the nearest aspect of the ablation zone, but actually obscures the deep border of the ablation (Fig. 6). Therefore, to overcome these limitations of US-guided percutaneous cryoablation, we performed repeated cryoablation by repositioning the cryoprobe during the procedure or performing a second or third session of cryoablation to achieve adequate ablation.
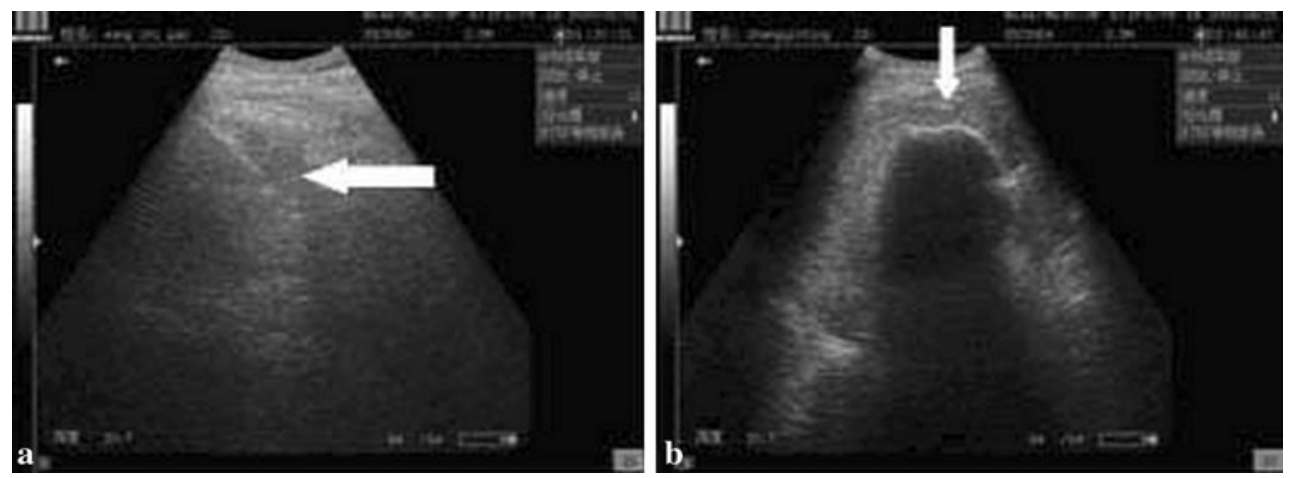

Fig. 6 Ultrasound (US) visualization of cryoprobe and frozen tissue during cryoablation. a US image obtained during placement of a cryoprobe into hepatocellular carcinoma (HCC). A longitudinal view of the cryoprobe is shown (arrow). The angle of approach and depth of the lesion are easy to estimate in the percutaneous setting and can help to position multiple cryoprobes. b Intraoperative US image of the to evaluate fully
Tumor location is another factor associated with local tumor recurrence. In our study, $61.2 \%$ of the tumors were close to the inferior vena cava or another large vessel. In such cases, care must be taken when performing cryoablation. Because of the heat-sink effect, nonlethal isotherms at the edge of the frozen tissue may affect blood vessel integrity $[16,17]$. Therefore, the probe should be located at a sufficient distance from the blood vessel. Unfortunately, this may mean an adequate ablation beyond the tumor border cannot be achieved.

Another interesting finding related to local recurrence involves the method of therapy. In our study, local recurrence was significantly higher in patients with incomplete ablation followed by TACE than in those with complete cryoablation, which differs from the results of previous studies [18, 19]. In fact, most of the data published on local ablation combined with TACE showed a decreased rate of recurrence and prolonged disease-free survival with this approach [19]. Even though this factor was excluded from the multivariate analysis because of the influence of tumor size, we should pay close attention to this phenomenon for the following reasons. First, TACE is a non-curative therapy. Although TACE has been reported to induce extensive tumor necrosis in $>50 \%$ of patients with an objective response ranging from 16 to $60 \%$, fewer than $2 \%$ of patients treated with TACE alone achieved a complete response [20, 21]. Second, the order of cryoablation or TACE may influence clinical outcomes. Combined strategies, incorporating ablation with TACE for the treatment of HCC, are likely to play a greater role in the future. However, no studies have prospectively compared the effect of performing ablation before or after TACE. On the other hand, a randomized controlled trial reported median survivals of 37,24 and 22 months, and complete tumor

frozen tissue during cryoablation of an HCC. The hyperechoic line represents the proximally edge of the frozen tissue (arrow). The posterior aspects of the frozen tissue are represented by an acoustic shadow. Although the contralateral side of the frozen tissue can be evaluated by insonating from a different angle, this region is difficult 

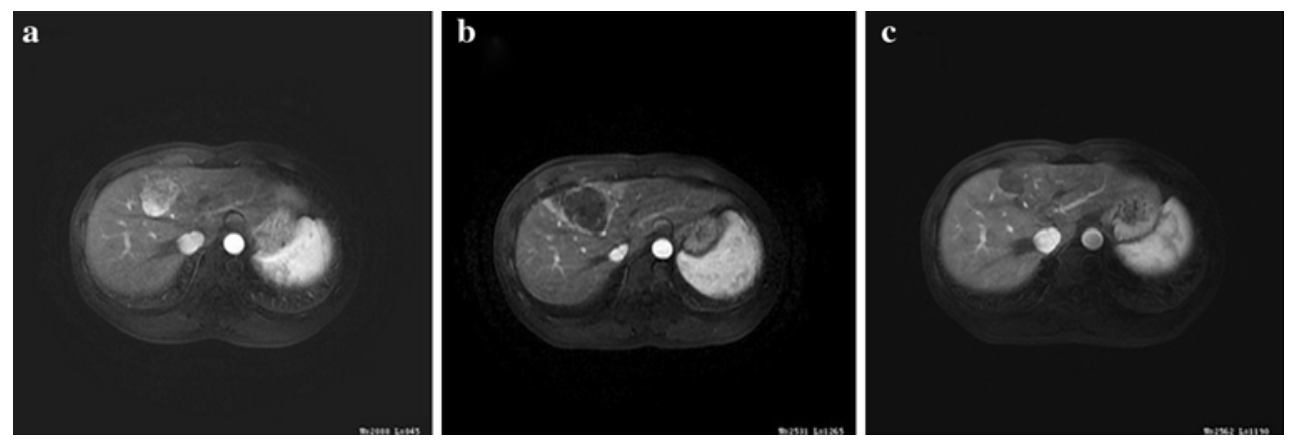

Fig. 7 MRI images (early phase) before and after treatment in a completely ablated HCC case. a MRI shows an HCC lesion with $4.2 \mathrm{~cm}$ in diameter before cryoablation. b MRI scanning at 1 week after cryoablation of HCC. The HCC lesion was completely ablated

response rates of 55, 5 and $37 \%$ for TACE followed by RFA, TACE alone and RFA alone, respectively [19]. In our study, the tumors were treated by cryoablation first, followed by TACE. In theory, performing cryoablation first can reduce the tumor load and thus enhance the effects of TACE. However, cryoablation can destroy the blood vessels supplying the tumor or create arterioportal or arteriovenous fistulas, thus limiting the potential efficacy of TACE [16]. These aspects need to be studied in more detail.

Complications of varying degrees of severity occurred during and after hepatic cryoablation. Although the majority were minor, severe complications occurred in 19 patients $(6.3 \%)$. We found that, in five patients with liver bleeding and one patient with intestinal fistulas, the ablated tumors were located in the subcapsular region, but were not surrounded by liver parenchyma. Tumors in a subcapsular location and tumors adjacent to the gallbladder or loops of the bowel can be problematic and should be treated with caution. The postoperative period is an important aspect of patient care as we may occasionally find life-threatening delayed hemorrhage during this time, and TAE should be done as soon as possible. In most cases, however, there were no true contraindications to ablation. However, seven patients with Child-Pugh class B (score $>8$ ) and TEA of cryoablation of $70 \%$ experienced severe liver damage. Two of these patients developed liver failure and died. Therefore, borderline liver dysfunction and TEA should be considered, and may present contraindications to cryoablation. Cryoshock is a severe systemic adverse effect that has been reported to occur in up to $1 \%$ of all hepatic cryoablations $[22,23]$. This disorder may be related to the ablation zone reperfused and resorption of necrotic debris directly into the bloodstream during cryoablation [16, 22]. In our series, only six patients developed mild cryoshock, and all recovered rapidly following intravenous administration of atropine and 5\% sodium bicarbonate, and warming during the cryoablation. showing a hypovascular zone with a hypervascular inflammatory rim around the ablation zone. c MRI scanning at 12 months after treatment

In this study, the overall 1-, 2- and 3-year survival rates were 80,45 and $32 \%$, respectively, with a mean survival of 28 months and a median survival of 22 months. For patients with early HCC (i.e., one to three HCCs of $<3 \mathrm{~cm}$ in diameter), the 1-, 2-, 3- and 5-year survival rates were $91,85,65$ and $54 \%$, respectively. Tumor stage is an important prognostic factor for the long-term outcomes of cryoablation. Most of the intermediate and advanced HCCs were combined with a poor Child-Pugh classification, large tumor size and intra- or extrahepatic metastasis, which resulted in low survival rates. In our study the median overall survival (OS) of the advanced HCC was 8.6 months. For comparison, the median OS of similar patients was only 4 months and 6.5 months in advanced HCC patients receiving combination local therapy and systemic treatment. The encouraging results suggest that patients with advanced HCC may benefit from cryoablation of HCC. This deserves further study. Analysis of causes of death in our study revealed that the leading cause was esophagogastric variceal bleeding due to portal hypertension, accounting for $36.3 \%$ of all deaths. Liver failure was the second most common cause of death, accounting for $26.2 \%$ of all deaths, while tumor recurrence and metastasis accounted for $23.2 \%$ of deaths. These findings indicate that prevention of esophagogastric variceal bleeding in liver cirrhosis and maintaining good liver function can avoid up to $62 \%$ of deaths.

During follow-up, contrast-enhanced CT scanning or MRI and alpha feto-protein (AFP) determination every 2 months after the cryoablation are crucial for detection of tumor recurrence. One imaging feature that can complicate the short-term postablation imaging evaluation is a hypervascular inflammatory rim that develops around the ablation zone. It appears about 1-2 weeks after the cryoablation and disappear 2 weeks later, and is not regarded as tumor recurrence (Fig. 7).

In conclusion, this study revealed that percutaneous cryoablation is a relatively safe and effective therapy for 
HCC. We believe this approach is best suited for regional tumors of $<5 \mathrm{~cm}$ in diameter. Postoperative management is very important for timely detection of delayed hemorrhage. With improvements in technology and advances in cryoprobes and guidance, percutaneous cryoablation represents a robust ablation modality and should be strongly considered for HCC and possibly other cancers, particularly when a precise or large zone of ablation is required.

Acknowledgments This study was supported by the Key Scientific and Technological Research Foundation of the National Specialpurpose Program (2008ZX10002-018), and the Capital Medical Research and Development Fund (2009-2041).

Conflict of interest The authors declare that they have no conflicts of interest.

Open Access This article is distributed under the terms of the Creative Commons Attribution Noncommercial License which permits any noncommercial use, distribution, and reproduction in any medium, provided the original author(s) and source are credited.

\section{References}

1. Parkin DM. Global cancer statistics in the year 2000. Lancet Oncol. 2001;2:533-43.

2. Tang ZY. Perspective of clinical oncology from the viewpoint of liver cancer studies. Tumor (China). 2009;29:1-4.

3. Poon RT, Fan ST, Lo CM, Ng IO, Liu CL, Lam CM, et al. Improving survival results after resection of hepatocellular carcinoma: a prospective study of 377 patients over 10 years. Ann Surg. 2001;234:63-70.

4. Orlacchio A, Bazzocchi G, Pastorelli D, Bolacchi F, Angelico M, Almerighi $\mathrm{C}$, et al. Percutaneous cryoablation of small hepatocellular carcinoma with US guidance and CT monitoring: initial experience. Cardiovasc Intervent Radiol. 2008;31:587-94.

5. Atwell TD, Farrell MA, Callstrom MR, Charboneau JW, Leibovich BC, Patterson DE, et al. Percutaneous cryoablation of 40 solid renal tumors with US guidance and CT monitoring: initial experience. Radiology. 2007;243:276-83.

6. Lee FT Jr, Mahvi DM, Chosy SG, Onik GM, Wong WS, Littrup PJ, et al. Hepatic cryosurgery with intraoperative US guidance. Radiology. 1997;202:624-32.

7. Shock SA, Laeseke PF, Sampson LA, Lewis WD, Winter TC 3rd, Fine JP, et al. Hepatic hemorrhage caused by percutaneous tumor ablation: radiofrequency ablation versus cryoablation in a porcine model. Radiology. 2005;236:125-31.
8. Llovet JM, Brú C, Bruix J. Prognosis of hepatocellular carcinoma: the BCLC staging classification. Semin Liver Dis. 1999;19:329-38.

9. Wang C, Lu Y, Chen Y, Feng Y, An L, Wang X, et al. Prognostic factors and recurrence of hepatitis B-related hepatocellular carcinoma after argon-helium cryoablation: a prospective study. Clin Exp Metastasis. 2009;26:839-48.

10. Zuro LM, Staren ED. Cryosurgical ablation of unresectable hepatic tumors. AORN J. 1996;64(231-236):239-44.

11. Sabel MS. Cryo-immunology: a review of the literature and proposed mechanisms for stimulatory versus suppressive immune responses. Cryobiology. 2009;58:1-11.

12. Osada $S$, Imai $H$, Tomita $H$, Tokuyama $Y$, Okumura $N$, Matsuhashi N, et al. Serum cytokine levels in response to hepatic cryoablation. J Surg Oncol. 2007;95:491-8.

13. Shimizu T, Sakuhara Y, Abo D, Hasegawa Y, Kodama Y, Endo H, et al. Outcome of MR-guided percutaneous cryoablation for hepatocellular carcinoma. J Hepatobiliary Pancreat Surg. 2009;16: 816-23.

14. Nagata Y, Hiraoka M, Nishimura Y, Masunaga S, Mitumori M, Okuno Y, et al. Clinical results of radiofrequency hyperthermia for malignant liver tumors. Int J Radiat Oncol Biol Phys. 1997;38:359-65.

15. Helling TS. Realistic expectations for cryoablation of liver tumors. J Hepatobiliary Pancreat Surg. 2000;7:510.

16. Hinshaw JL, Lee FT Jr. Cryoablation for liver cancer. Tech Vasc Interv Radiol. 2007;10:47-57.

17. Kerkar S, Carlin AM, Sohn RL, Steffes C, Tyburski J, Littrup P, et al. Long-term follow up and prognostic factors for cryotherapy of malignant liver tumors. Surgery. 2004;136:770-9.

18. Xu KC, Niu LZ, Zhou Q, Hu YZ, Guo DH, Liu ZP, et al. Sequential use of transarterial chemoembolization and percutaneous cryosurgery for hepatocellular carcinoma. World J Gastroenterol. 2009;15:3664-9.

19. Cheng BQ, Jia CQ, Liu CT, Fan W, Wang QL, Zhang ZL, et al. Chemoembolization combined with radiofrequency ablation for patients with hepatocellular carcinoma larger than $3 \mathrm{~cm}$ : a randomized controlled trial. JAMA. 2008;299:1669-77.

20. Llovet JM, Bruix J. Systematic review of randomized trials for unresectable hepatocellular carcinoma: Chemoembolization improves survival. Hepatology. 2003;37:429-42.

21. Bruix J, Sala M, Llovet JM. Chemoembolization for hepatocellular carcinoma. Gastroenterology. 2004;127(5 Suppl 1):S179-88.

22. Seifert JK, Morris DL. World survey on the complications of hepatic and prostate cryotherapy. World J Surg. 1999;23:109-13.

23. Ng KK, Lam CM, Poon RT, Shek TW, To JY, Wo YH, et al. Comparison of systemic responses of radiofrequency ablation, cryotherapy, and surgical resection in a porcine liver model. Ann Surg Oncol. 2004;11:650-7. 